\title{
The small world of stakeholder groups: cascading concepts for urban change
}

\author{
M. Ganis, J. Minnery \& D. Mateo-Babiano \\ The University of Queensland, School of Geography, \\ Planning and Environmental Management, Australia
}

\begin{abstract}
This research seeks to understand why some stakeholder groups adopt novel urban design notions and others tend to lag in the uptake of these ideas. Key stakeholder groups participated in a semantic differential task of bipolar urban design qualities. A concept mapping task revealed those groups who aligned in their concept of good urban places and by implication, those that differed. The results showed that architects and planners are early adopters of urban design concepts, engineers, developers and the community are an early majority of adopters and landscape architects and councillors are the late majority. A small world metaphor offers a model for the spread of ideas between groups.

Keywords: perception of place, small world networks.
\end{abstract}

\section{Introduction}

Urban change is sometimes fraught with difficult planning negotiations. Such difficulties occur because stakeholders' planning expectations are not often satisfied. We need to elicit an underlying conceptual structure of their expectations for urban places. This discussion will consider firstly: the perception of place as a cognitive process; the role of small world network theory in understanding the process of the spread of ideas; and finally, the results of an empirical study interpreted in the context of a small world approach in understanding stakeholder groups' acceptance of novel ideas in planning urban change. 


\section{Adaptive perceptual cycle}

In planning for urban change we need to understand stakeholders' perception of the urban context. The Adaptive Perceptual Cycle describes how people perceive their world and adapt to changes in it [1-4]. The Adaptive Perceptual Cycle (Figure 1) illustrates how we experience a place guided by existing expectations (or schemata). Information is absorbed as we experience a place (physical, social, cultural etc.) that tests our expectations or schemata. This contextual information may either 'fit' our schema or conflict with it. If our schema is not a 'good fit' a crisis of relevance occurs and a choice must be made to either adapt or not. This is the point of criticality [2] whereby the schema either resists change (systemic rigidity) or adjusts and adapts to the new context (systemic shift).

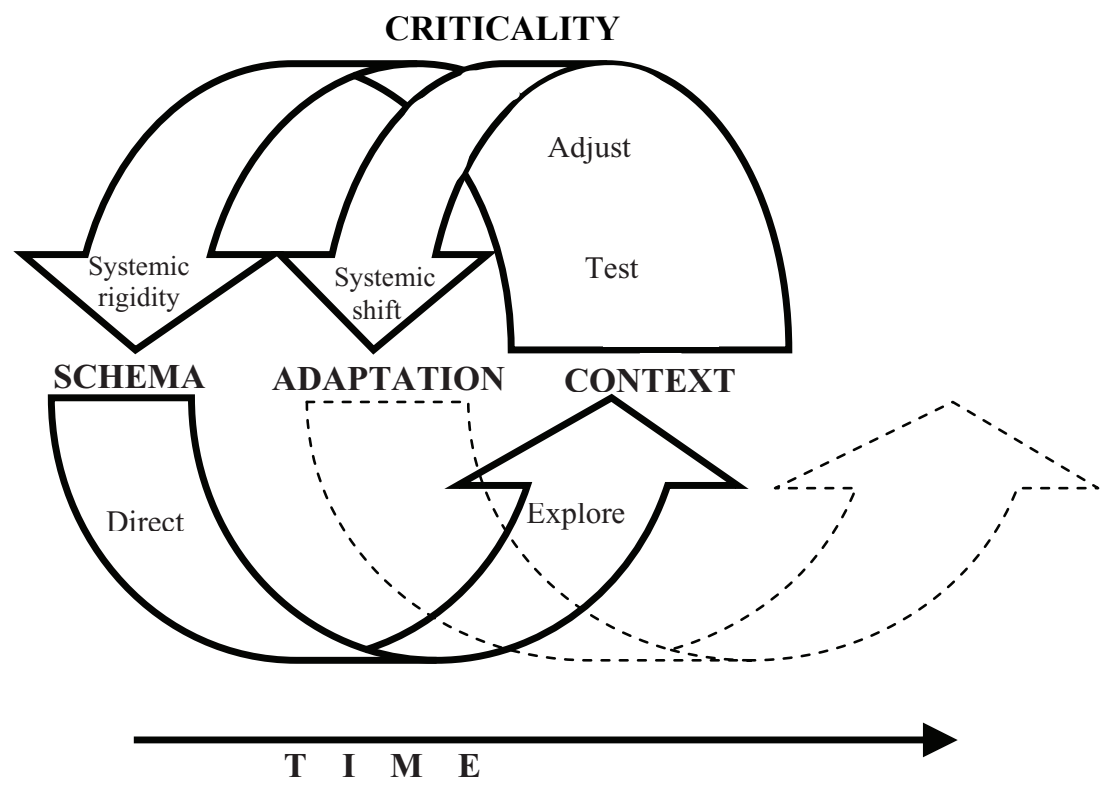

Figure 1: $\quad$ Adaptive Perceptual Cycle [1-4].

Resistance to change threats the stability of a whole system - small local instabilities engulf a whole system (schema) and threaten global instability (criticality) creating a change imperative (self organisation). This self organising change in the Adaptive Perceptual Cycle is its point of criticality [3]. Synergetics (from the Greek word meaning, 'working together') contributes to the idea of self-organising adaption. Consider the complex network of factors that make up an urban context (these might be political, economic, social etc.). These factors present order parameters (such as regulations, policies, traditions etc.) which help structure personal and social knowledge structures. Order parameters 
compete to capture control of a knowledge structure. As Haken puts it, the order parameter that 'captures' and 'enslaves' its competitors wins [5]. The point of 'enslavement' represents a critical shift or a phase transition. Just as synergetics captures ideas until dominant concepts emerge, the Adaptive Perceptual Cycle tests concepts until a point of criticality demands adaptation.

In an urban context, our knowledge structure is challenged by many order parameters from culture to politics; and pressures from population to climate change. Many powerful protagonists such as developers, politicians, economists, environmentalists, design professionals, community and so on contest an urban change context. A network of ideas frames their knowledge structure of the urban concepts with which they grapple. Here, this network of urban design ideas is plotted as a concept map and interpreted using network typology.

\section{Network typology}

A network is a relationship of vertices (nodes or points) and edges (links or connectors). Traditionally, network topology identifies the stability of a regular network and the instability of a random network Figure 2). Within the last decade or so, a middle way between regular and random networks has emerged termed 'small world' networks derived from the Milgram's sociological notion popularly known as 'six degrees of separation' [7]. These 'degrees' are the connections across social groups that expedite our social affiliations purportedly an average of six affiliates between any two people on the planet - this is the 'small world'.
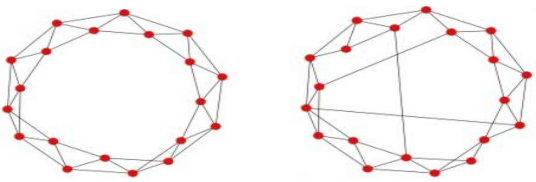

WS small world model Watts and Strogatz (1998)
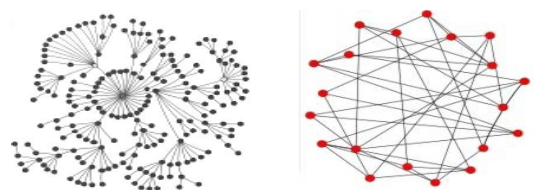

BA scale free model
REGULAR

$$
\text { SMALL WORLD (2 types) }
$$

\section{RANDOM}

\section{Figure 2: $\quad$ Network topology $[8,9]$.}

A small world network is one that combines the characteristics of a regular, ordered network with those of a random, flexible one. Regular networks are characteristically coherent, but slow to change. Random networks are incoherent, but change rapidly. A small world network has the stability of coherence as well as the flexibility of rapid adaptation to change $[8,9]$.

To begin at the beginning, imagine living in a caveman world (Figure 2). In each cave lives a group of cavemen who only know each other and no one else. One curious caveman ventures out and meets another caveman passing by: "their propensity to be acquainted immediately becomes very high and stays that way 
regardless of how many additional mutual friends they may have" [6]. Now that our caveman world is connected, imagine a social gathering during which one caveman randomly meets another and on discovery of a mutual acquaintance exclaims "Small world isn't it!". This mutual acquaintance is a random, longrange 'short cut' between formerly distant clusters of cavemen. Affiliations via such 'short cut' acquaintances beyond one's own cave significantly increases the import of novel ideas and innovations, which may challenge the stability of traditional concepts or schemata.
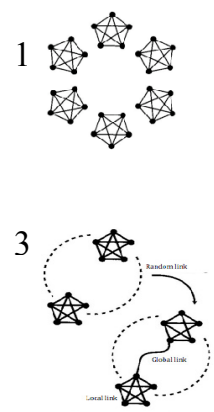

2

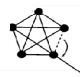

4

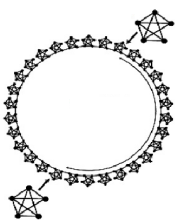

Figure 3: $\quad$ Watts' caveman world [6].

A small world network combines the stability of a regular network and the creativity of a random one. If we apply this small world network metaphor to the Adaptive Perceptual Cycle this represents how schemata are enriched and changed by novel ideas and innovation. We test this new schema in the real world until the real world experience no longer 'fits' that schema. New information arises that triggers a battle of order parameters and eventually impels a cascade of change.

\section{Cascades, thresholds and the spread of ideas}

In the context of the Adaptive Perceptual Cycle the threshold is the level of criticality and the cascade represents the systemic shift of a concept. Cascades are a characteristic dynamic of complex systems. Cascades appear as critical failures in many complex systems from built form infrastructure to the progression of climate change. The structure of such complex systems withstands many shocks over time and yet can fail unpredictably. This suggests that the structure of complex systems is ambiguously robust yet fragile [11-13]. Is it the structure of the system or the intensity of the shock that impels a cascade of change?

Gladwell [10] purports that a special person triggers a cascade of change such as "shocking!" Hollywood starlets who trigger cascades of changing fads and fashions. Watts [14] and Watts and Dodds [25] counter the special person argument with this analogy - the spark that ignites a forest fire is no different from any other spark. A forest fire is fuelled by a combination of temperature, 
wind and vegetation - it is the structure of the forest system rather than the quality of the spark that triggers a forest fire. The difference is that Gladwell's world is steered by the star-power of a principal actor $[10,15]$ whereas Watts' world is a constellation of random rocks and gases.

Thresholds set a system's level of robustness or fragility. Some people have a high threshold of change (robust) and align with convention. Those with a low threshold of change (fragile) willingly align with unconventional people. This means that in any given social group for any given fad the timing of cascades will differ - some people are early adopters, some are the early majority, some are the late majority and some are laggards [16-18].

The first step in the adoption of a novel idea is the awareness of its advantages or 'good fit' with an existing concept. Novel ideas that are compatible with existent schemata offer a familiar, stable conceptual framework from which to explore an unfamiliar or complex concept. Conversely, a 'bad fit' idea is likely to be rejected. Finally, adoption of a novel idea is evaluated through testing and observation of how successful the novelty is. Success observed or reported by others drives a novel idea via the few early adopters, the early majority who popularise a novelty and eventually spread it to the late majority and finally, possibly to the few laggards $[16,17]$.

Novelty needs an innovator. The innovator is the one who ventures forth out of their conceptual 'cave' and initially broadcasts the seed of a novel idea [11, 16]. Fertile soil is found in a low threshold context in which new ideas are compatible with the schemata of susceptible individuals. Early adopters are open to novelty particularly if there is an expectation from their colleagues that they are somewhat avant-garde and creative. If this group of susceptible early adopters is a homogeneous, like-minded close-knit group the novelty is rapidly adopted. However, if a homogeneous group has such strong ties that they know only each other and no one else - much like an isolated caveman - the novelty remains predominantly within the group and trickles slowly throughout the wider community.

Conversely, a heterogeneous group exposes a novel idea to diverse people with different interests connecting them beyond the group's conceptual 'cave'. This heterogeneous group of lesser-like-minded people have diverse acquaintances (weak ties) and act like a conduit for the spread of ideas [19-22]. The 'strength of weak ties' is that they generate a cascade of early majority adopters via their tacit connections with affiliates of other diverse groups $[6,16]$.

An early majority is a mass movement of followers that spreads novelty. The early majority generates the acceptance of a novel idea because the more people are attracted to a following the more people follow. Early majority cascades demonstrate a level of certainty that a novelty is tried and tested, acceptable and popular. This reassures the high level threshold that the risk-averse late majority needs before adopting a novel idea. And finally, the small group described as laggards [16-18] are those who adhere fiercely to conventional ideas despite the cascades of change around them. They resist the external shocks that persistently test their schemata of the world. Some may retreat into isolation and others may 
form exclusive communities who adhere to age-old ideas and practices and are impervious to the new.

Network structure influences the global spread of novel ideas [11] [26]. The points (vertices) of small world networks represent 'adopters' and the lines (connectors) represent the 'ties' between adopters. There are two types of small world networks (Figure 2). The first is the Watts and Strogatz [8] WS small world network model and the second is the Barabasi and Albert [9] BA scale-free network. The difference between these two small world models is the connectivity distribution, the growth mechanism and the composition. The key similarities that characterise both as small world networks are that they exhibit dense clusters, shortest path connectivity and self-organising evolution over time.

The WS small world network is a homogeneous model in which 'adopters' connect with their nearest neighbour $[8,21,23,24]$. It is an exponential growth model that tends towards a sparse distribution of dense local clusters (hubs) connected via long range short-cuts to the global network - somewhat like road network of towns and cities. The BA scale-free network is a heterogeneous model in which 'adopters' exhibit preferential attachment connecting with the rich and powerful - a 'rich get richer' power law distribution mechanism [9]. The BA model forms a densely connected giant component (hub) that dominates the global connectivity of the network - somewhat like an airline network of major airports around the world.

Network robustness and fragility are affected by the different structure of small world networks [11-13]. A WS small world model is a homogeneous network in which the low threshold of like-minded colleagues creates cascade fragility enabling the early adoption of ideas. However, the fragility of the cluster of early adopters does not affect the robustness of the WS small world network because of the sparse distribution of clusters - ideas will cascade incrementally. The BA scale free model may have the robustness of heterogeneity but the network is dominated by a giant component to which most adopters attach. A targeted attack on this dominant hub fragments the whole network and ideas will cascade catastrophically.

\subsection{Method}

Empirical case studies of small world networks have been described for many real world contexts but as yet, research into psychological networks is limited [29]. The intent of the concept mapping task is to reveal the stakeholders' schemata of urban design qualities that make good urban places. Multidimensional scaling (MDS) is a relational statistical method traditionally used in psychology and social network analysis [15, 27]. Cluster Analyses (CA) support the interpretation of the MDS plots. If there is ambiguity between the MDS and the CA the MDS result takes precedence [28]. A Kendall Tau correlation elicits those groups whose schemata are similar and those that are different.

A semantic differential task presents bipolar categories of urban design qualities derived from a content analysis of the urban design literature. The participant groups consist of a stratified random selection of design, development, community professionals and a lay group. These stratified groups 
are selected because they represent the usual stakeholders in an urban change process: Architects (10); Councillors (elected local government representatives) (8); Developers (7); Landscape Architects (7); Planners (24); Transport Engineers/Planners (11); and, Others (9) a total of 76 participants. The participants are instructed to respond from the perspective of their professional or community role. The urban change scenario is described so that their responses are framed by a real world context with which they are familiar South East Queensland, Australia.

"The predicted growth in South East Queensland over the next 20 - 25 years will trigger changes for existing urban centres. These changes will have an impact upon the urban design qualities of urban centres. Please mark with an $X$, the ranking number that best fits your idea of good urban design qualities for South East Queensland." The bipolar categories of urban qualities that the participants rank (from 1-7) are:

Table 1: $\quad$ Coding and bipolar categories for semantic differential task.

\begin{tabular}{|c|l|}
\hline Var. 1 & Compact, Distinct Centres - Dispersed Sub-centres \\
Var. 2 & Local Character Building Style - Unusual, Landmark Architecture \\
Var. 3 & Clear Way-finding - Mysterious Exploration \\
Var. 4 & Technical Efficiency - Sensory Experience \\
Var. 5 & Small-scale Local Infrastructure - Large-scale Regional Infrastructure \\
Var. 6 & Adventurous Places - Safe Places \\
Var. 7 & Urban-Rural Fusion - Ecological Conservation \\
Var. 8 & Evolving Places - Completed Places \\
Var. 9 & Movement Network Connectivity - Movement Network Separation \\
\hline
\end{tabular}

\subsubsection{Results}

The MDS plots consist of clusters of variables (shown as groups of dots) and dimensions (shown as a line or curve of dots). The plots are two-dimensional for clear interpretation. The stress value of an MDS analysis is an evaluation the results' 'goodness-of-fit': a stress value of $<0.15$ is a 'good fit' and one that is $>0.25$ is a 'bad fit'. A low stress value indicates greater reliability and a high the stress value indicates less reliability because the results may be too complex to interpret.

The results indicate a range of stress values for each group between $>0.01$ and $<0.16$ - this is a 'good fit'. Interpreting MDS plots is essentially a qualitative process [15] and the CA supports the interpretation of clusters, dimensions and Euclidean distance of the network of variables [29]. Although the MDS results show that each group's plot of their network or urban design variables is different there is one compelling relationship of variables - the All Groups plot (76 participants) illustrates the cluster of: Variable 1 (Compact, Distinct Urban Centres); Variable 3 (Clear Way finding); and, Variable 9 (Movement Network Connectivity). This group of variables is categorised as Cluster and Connectivity.

A Kendall Tau correlation analysis (Table 2) reveals groups that have similar conceptual structures and those that do not. Briefly, a co-efficient that is nearest 1 indicates the groups that are most closely related (a perfect correlation being 1) 
Table 2: $\quad$ Kendall Tau correlations MD pairwise deleted.

\begin{tabular}{|c|c|c|c|c|c|c|c|c|}
\hline \multicolumn{7}{|c|}{ Marked correlations (shown in bold) are significant at $\mathrm{p}<.05000$} \\
\hline & $\mathrm{ALL}$ & $\mathrm{A}$ & $\mathrm{C}$ & $\mathrm{D}$ & $\mathrm{LA}$ & $\mathrm{P}$ & $\mathrm{T} / \mathrm{P}$ & $\mathrm{O}$ \\
\hline $\mathrm{ALL}$ & 1.000 & $\mathbf{0 . 9 4 4}$ & 0.309 & $\mathbf{0 . 7 4 3}$ & $\mathbf{0 . 7 0 4}$ & $\mathbf{1 . 0 0 0}$ & $\mathbf{0 . 8 1 6}$ & $\mathbf{0 . 8 5 7}$ \\
\hline $\mathrm{A}$ & $\mathbf{0 . 9 4 4}$ & 1.000 & 0.253 & $\mathbf{0 . 8 0 0}$ & $\mathbf{0 . 6 4 7}$ & $\mathbf{0 . 9 4 4}$ & $\mathbf{0 . 7 6 0}$ & $\mathbf{0 . 8 0 0}$ \\
\hline $\mathrm{C}$ & 0.309 & 0.253 & 1.000 & 0.289 & $\mathbf{0 . 5 1 4}$ & 0.309 & 0.342 & 0.434 \\
\hline $\mathrm{D}$ & $\mathbf{0 . 7 4 3}$ & $\mathbf{0 . 8 0 0}$ & 0.289 & 1.000 & $\mathbf{0 . 6 6 6}$ & $\mathbf{0 . 7 4 3}$ & $\mathbf{0 . 5 7 9}$ & $\mathbf{0 . 7 0 5}$ \\
\hline LA & $\mathbf{0 . 7 0 4}$ & $\mathbf{0 . 6 4 7}$ & $\mathbf{0 . 5 1 4}$ & $\mathbf{0 . 6 6 6}$ & 1.000 & $\mathbf{0 . 7 0 4}$ & $\mathbf{0 . 5 1 4}$ & $\mathbf{0 . 7 8 2}$ \\
\hline $\mathrm{P}$ & $\mathbf{1 . 0 0 0}$ & $\mathbf{0 . 9 4 4}$ & 0.309 & $\mathbf{0 . 7 4 3}$ & $\mathbf{0 . 7 0 4}$ & 1.000 & $\mathbf{0 . 8 1 6}$ & $\mathbf{0 . 8 5 7}$ \\
\hline TE/P & $\mathbf{0 . 8 1 6}$ & $\mathbf{0 . 7 6 0}$ & 0.342 & $\mathbf{0 . 5 7 9}$ & $\mathbf{0 . 5 1 4}$ & $\mathbf{0 . 8 1 6}$ & 1.000 & $\mathbf{0 . 7 2 4}$ \\
\hline $\mathrm{O}$ & $\mathbf{0 . 8 5 7}$ & $\mathbf{0 . 8 0 0}$ & 0.434 & $\mathbf{0 . 7 0 5}$ & $\mathbf{0 . 7 8 2}$ & $\mathbf{0 . 8 5 7}$ & $\mathbf{0 . 7 2 4}$ & 1.000 \\
\hline
\end{tabular}

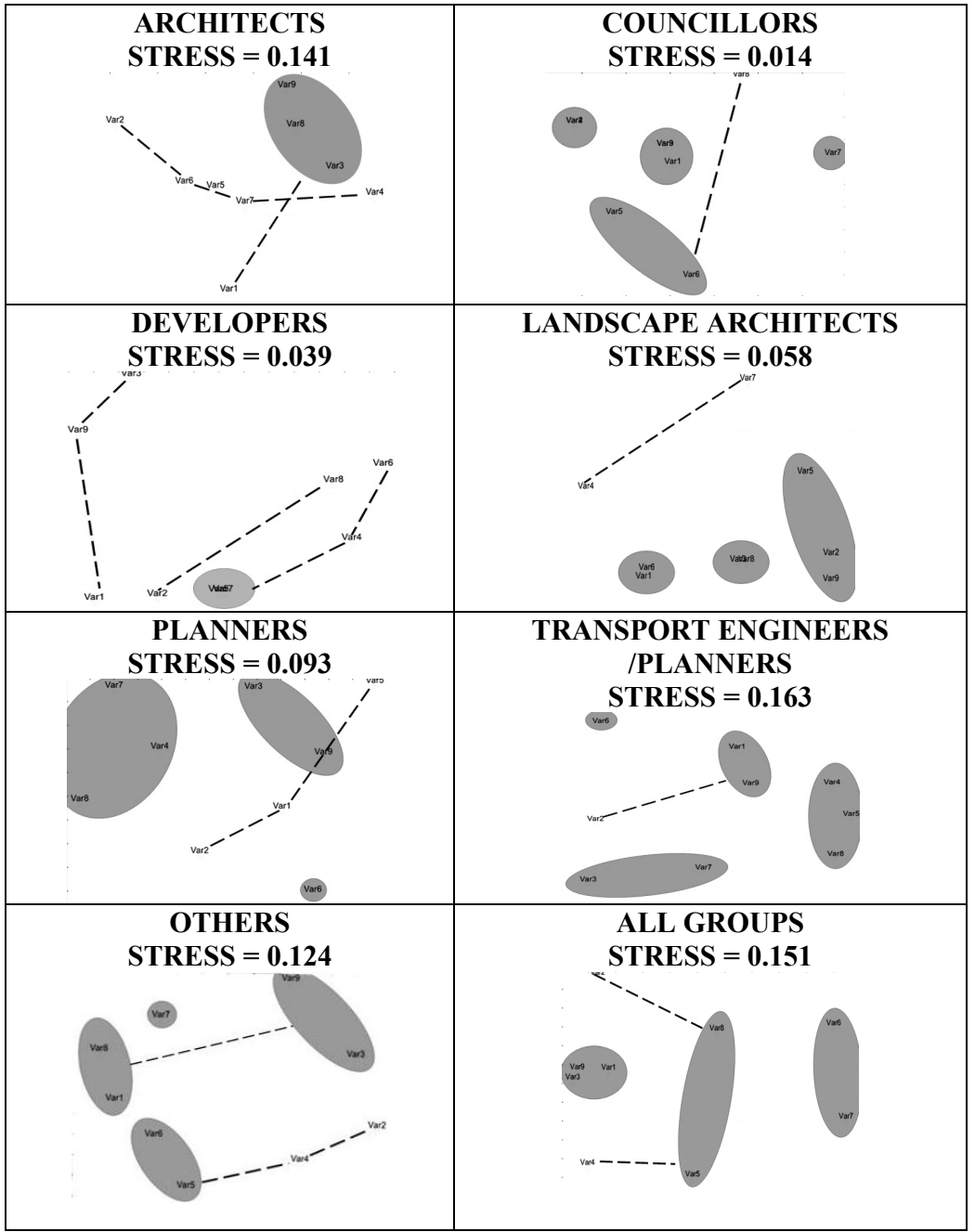

Figure 4: $\quad$ Multidimensional scaling plots. 
in their conceptual structure of good urban places, and those furthest, less related. The results show that the only group that has some correlation with the Councillors (C) is the group of Landscape Architects (LA) with a coefficient of 0.5 . The groups with a coefficient indicating a similar conceptual structure are the Architects (A) and the Planners (P) with a coefficient of 0.94. The groups that are least correlated with the Councillors (C) are the Architects (A) with a coefficient of 0.25, the Developers (D) with a coefficient of 0.29 and the Planners (P) with a coefficient of 0.31 .

\subsection{Discussion}

The pressures of urban growth and change in South East Queensland, Australia triggered the research. Planning for dynamic urban change is difficult because stakeholders' expectations often differ. This research sought to reveal the similarities and differences between stakeholders' schemata of good urban places. A concept mapping task was intended to reveal the content and structure of the stakeholders' schemata. Multidimensional scaling analysis (MDS) plotted the stakeholders' concept maps supported by cluster analyses (CA) and Kendall Tau correlations. The interpretation of the concept maps was framed within a small world network metaphor.

Firstly, consider the All Groups MDS plot: Variable 1 (Compact, Distinct Urban Centres); Variable 3 (Clear Way finding); and, Variable 9 (Movement Network Connectivity). Interpreted with a small world metaphor in mind, this cluster of variables represented a hub of the participants' schema - a component of the network categorised in this discussion as urban Cluster and Connectivity.
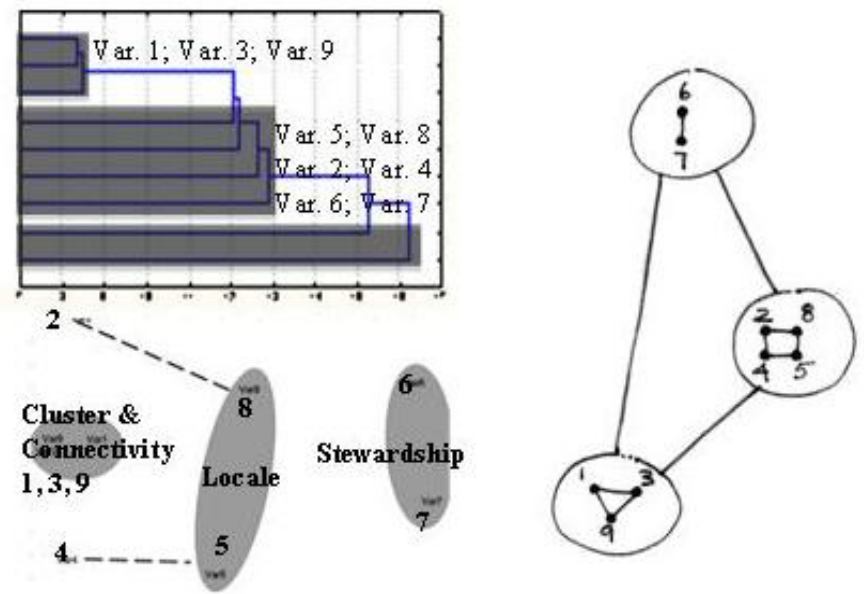

Figure 5: All Groups cluster analysis, MDS plot and small world diagram.

Cluster and Connectivity formed a hub that may represent a schema of urban density connected throughout by an efficient and coherent street network. Interestingly, the variables of this hub - Cluster and Connectivity - also describe 
the dynamic of small world networks whether a WS small world exponential model or a BA scale free preferential attachment model. This implies that a small world network dynamic not only emerged in the content of the schema (i.e. the variables categorised as Cluster and Connectivity) but also as the dynamic of network structure.

The adjacent cluster of variables consisted of: Var. 5 Small-scale Local Infrastructure; Var. 8 Evolving Places; Var. 2 Local Character Building Style; and, Var. 4 Sensory Experience. This hub was categorised as Locale because the variables described the urban setting in both concrete and abstract terms. Locale is the nearest neighbour to the Cluster and Connectivity hub suggesting suggests that the urban form of Cluster and Connectivity is associated with stakeholders' experience of the Locale.

The hub that consisted of: Var. 6 Safe Places; and, Var. 7 Ecological Conservation is defined as Stewardship. It was the least contiguous with the hub Cluster and Connectivity however, the nearest neighbour to the hub Stewardship is Locale. This implies that the notion of Locale is associated with a sense of Stewardship for the safety of people and the environment.

The results of the Kendall Tau correlations showed that the most similar stakeholder groups were the Architects and Planners. Conversely, the Councillors were the least similar and only somewhat similar to the Landscape Architects. These two extremes presented a curious scenario. The role of Architects and Planners is to offer the conceptual input into a planning process and it was expected that the Landscape Architects would align with these design professionals. Counter intuitively, the Landscape Architects were the only group who had some similarity with the Councillors and of the professional groups they had the least similarity with the Architects and Planners. This significant correlation between the Landscape Architects and the Councillors can only be conjecture. It might be that Landscape Architects have a schema dominated by natural landscaping concepts and the Councillors may be comfortable such concepts as the restorative effects of vegetation, natural environments, parks and open spaces. This may be a strong conceptual tie between them whereas the other professionals may have a predominantly urban built form focus.

The Architects and Planners (and ignoring the puzzling Landscape Architects for the moment) are homogenous stakeholder groups with closely aligned education and experience with urban design notions. The familiar urban design schemata act as a stable construct enabling the early adoption of novel ideas. Early adopters of novel ideas have a low threshold (fragility) that triggers a cascade of change New ideas are adopted more readily if the systemic shift of the schema (cascade) is moderate rather than extreme.

Most of the remaining stakeholder groups revealed a less significant correlation with the early adopter Architects and Planners (0.944). Comparing each group's correlation coefficient with All Groups (1.000) the Architects and Planners were the early adopters; the Others $(0.857)$ and the Transport Engineers/Planners (0.816) were the early majority; and the late majority appeared to be the Developers (0.743) and the Landscape Architects (0.704). Finally, the Councillors appeared to be the laggards with an insignificant 
correlation coefficient of 0.309 . An argument may be posed that Councillors have many considerations to juggle other than urban design ones but this does not explain their correlation with the Landscape Architects nor does it explain the correlation between the diverse group of Others and the Architects and Planners. Why are the Others more similar with the design professionals (except the Landscape Architects) than the Councillors when both of these groups are generally lay groups and community representatives?

The Others consisted of a random group of lay people - a heterogeneous group. As Granovetter and others propose, their diversity offers random weak ties that are a strong conduit for the spread and receipt of novel ideas [19, 22]. Conversely, most professional groups are likely to be homogeneous which enables the early adopter cascade of novel ideas but also increases redundant contacts.

\section{Conclusion}

The Adaptive Perceptual Cycle described how schemata guide the way we interpret the world. Schemata cascade if novel ideas challenge existing expectations. Stakeholder groups' concept maps indicated that Cluster and Connectivity was a hub of their schema for good urban places. This aligned with small world network theory and may be a framework for understanding the robustness and fragility of stakeholders' concepts in planning urban places.

\section{Acknowledgements}

We thank Dr Phil Smith and Dr Mike Gillen, University of Queensland, Australia.

\section{References}

[1] Neisser, U. Cognition and Reality: principles and implications of cognitive psychology. New York: W. H. Freeman and Company, 1976.

[2] Portugali, J. (ed). The Construction of Cognitive Maps. London: Kluwer Academic Publishers, 1996.

[3] Bak, P. How nature works: the science of self-organized criticality. Oxford: Oxford University Press, 1997.

[4] Haken, H. and Portugali, J. The face of the city is its information. Journal of Environmental Psychology. Elsevier Ltd., 23 pp. 385-408, 2003.

[5] Haken, H. In Portugali, J. ed. The Construction of Cognitive Maps. London: Kluwer Academic Publishers, 1996.

[6] Watts, D. J. Small Worlds: the dynamics of networks between order and randomness. Princeton, NJ: Princeton University Press, p45, 1999.

[7] Milgram, S. "The Small World Problem”. Psychology Today. 1:1 pp. 60-67, 1967.

[8] Watts, D. J. and Strogatz, S. Collective dynamics of 'small world' networks. Nature. London: Macmillan Publishers, 393:4 pp. 440-442.1998. 
[9] Barabási, A.-L. and Albert, R. Emergence of Scaling in Random Networks. Science, 286, pp. 509-512, 1999.

[10] Gladwell, M. The Tipping Point: how little things can make a big difference. Boston: Little Brown, 2000.

[11] Watts, D. J. A simple Model of Global Cascades on Random Networks, 2002. In M. Newman, A-L Barabasi and D. J. Watts. The Structure and Dynamics of Networks. Princeton, NJ: Princeton University Press, 2006.

[12] Wang, X. F. and Chen G. Complex Networks: small world, scale free and beyond. IEEE Circuits and Systems. 2003.

[13] Sun, S., Liu, Z., Chen, Z. and Yuan, Z. Error and Attack Tolerance of Evolving Networks with Local Preferential Attachment. Physica A. Elsevier B. V. 2006.

[14] Watts, D. J. Challenging the Influentials Hypothesis. Measuring Word of Mouth, Dept. of Communication Studies, Northeastern University, vol. 3, 2007.

[15] Scott, J. Social Network Analysis: a handbook. London: SAGE Publications Inc., 2000.

[16] Rogers, E. M. Diffusion and Innovation. New York: Free Press, 1995.

[17] Valente, T. W. Social Network Thresholds in the Diffusion of Innovation. Social Networks. Elsevier B. V. 18, pp. 69-89.1996.

[18] Valente, T. W. Network Models and Methods for Studying the Diffusion of Innovations. In P.J. Carrington, J. Scott and S Wasserman. Models and Methods in Social Network Analysis. Cambridge: Cambridge University Press, 2005.

[19] Granovetter, M. The Strength of Weak Ties. American Journal of Sociology, 78:6 pp. 1360-1380, 1973.

[20] Granovetter, M. The Strength of Weak Ties: a network theory revisited. Sociological Theory. American Sociological Association, vol. 1 pp. 201-233, 1983.

[21] Kleinberg, J. M. "Navigation in a Small World". Nature. 406: 845, 2000.

[22] Watts, D. J. A simple Model of Global Cascades on Random Networks, 2002. In M. Newman, A-L Barabasi and D. J. Watts, The Structure and Dynamics of Networks. Princeton, NJ: Princeton University Press, 2006.

[23] Newman, M. E. J. and Park, J. Why Social Networks are Different from Other Types of Networks. Physical Review E, 68, 036122. The American Physical Society, 2003.

[24] Dodds, P. S. and Watts, D. J. Universal Behaviours in a Generalized Model of Contagion. Physical Review Letters. The American Physical Society 92:21, 2004.

[25] Watts, D. J. and Dodds P. S. Influentials, Networks and Public Opinion Formation. Journal of Consumer Research, vol. 34 pp. 441-458, 2007.

[26] Coronges, K. A., Valente, T. W. and Stacy, A. W. Structural Comparison of Cognitive Associative Networks in Two Populations, 2007. www.onlinelibrary. $\quad$ wiley.com/doi/10.1111/j.1559-1816.2007.00253.x/ abstract. 
[27] Wasserman, S. and Faust, K. Social Network Analysis: methods and applications. Cambridge: Cambridge University Press, 1994.

[28] Trochim, W.www.socialresearchmethods.net/mapping/mapping.htm

[29] Schnettler, S. A structured overview of 50 years of small-world research. Social Networks, 31, pp. 165-178, 2009. 